\title{
Seltene Ursache für eine Problematik des Sakroiliakalgelenks bei einem Ironman-Triathleten
}

\author{
Knechtle, Beat ; Nikolaidis, Pantelis T
}

DOI: https://doi.org/10.1024/1661-8157/a002586

Posted at the Zurich Open Repository and Archive, University of Zurich ZORA URL: https://doi.org/10.5167/uzh-137925

Journal Article

Originally published at:

Knechtle, Beat; Nikolaidis, Pantelis T (2017). Seltene Ursache für eine Problematik des Sakroiliakalgelenks bei einem Ironman-Triathleten. Praxis, 106(2):97-102.

DOI: https://doi.org/10.1024/1661-8157/a002586 
Gesundheitszentrum St. Gallen, Exercise Physiology Laboratory, Nikaia, Greece

Beat Knechtle, Pantelis T. Nikolaidis

Seltene Ursache für eine Problematik des Sakroiliakalgelenks bei einem Ironman Triathleten

Rare reason for a problem of a sacroiliac joint dysfunction in an Ironman triathlete 
Im Artikel verwendete Abkürzungen

$\begin{array}{ll}\text { LWS } & \text { Lendenwirbelsäule } \\ \text { MRI } & \text { Magnetresonanztomographie } \\ \text { SIG } & \text { Sakroiliakalgelenk }\end{array}$




\section{Anamnese und Befunde}

Der 52-jährige Ironman-Triathlet meldet sich in der Sprechstunde wegen seit mehreren Wochen anhaltender Schmerzen im linken Gesäss. Er berichtet dass er in der finalen Vorbereitung auf einen Wettkampf sei. Seit er sein Zeitfahrrad für den geplanten Ironman-Wettkampf einfahre und die linke Schuhplatte anders eingestellt habe zwicke es im Bereich des Gesässes links. Und wenn er auf der Bahn Intervalle laufe so bekomme er bei höheren Geschwindigkeiten immer mehr Schmerzen an der gleichen Stelle im Bereich des Beckens. Der Schmerz sei nicht schlimm, gehe aber auch nicht weg, störe weder beim Schwimmen noch beim Radfahren, aber schnellere und/oder längere Laufeinheiten seien wegen zunehmender Schmerzen nicht mehr möglich. Und das löse einen deutlichen Stress aus da ein Qualifikationsrennen für den Start an einem wichtigen Wettkampf im Herbst anstehe.

Im Status findet sich ein gut austrainierter Mann mittleren Alters ohne Wirbelsäulenfehlstellungen, Beckenschiefstand oder Beinlängendifferenz. Das Sakroiliakalgelenk (SIG) zeigt links eine Druckdolenz, einen positiven Vor- sowie einen verminderten Rücklauf, ein negatives Mennel-Zeichen, ein negatives ViererZeichen und die Diagnose eines hypomobilen SIG links wurde gestellt.

\section{Differentialdiagnostische Überlegungen}

Zum Zeitpunkt der Konsultation war der Verdacht auf eine Dysfunktion des linken SIG sehr gross zumal der klinische Untersuch sehr für diese Problematik sprach. Grundsätzlich wären bei einem Mann im mittleren Alter auch eine beginnende Coxarthrose sowie ein Problem der LWS durchaus möglich. Eine tumoröse Veränderung im Sinne eines ossären Primärtumors oder einer Metastasierung im Beckenskelett erschien sehr unwahrscheinlich ebenso wie eine rheumatische oder entzündliche Erkrankung bei einem gut trainierten Athleten.

\section{Weitere Abklärungsschritte und Verlauf}

Nach der ersten Manipulation probierte der Athlet wieder zu laufen, musste aber bei rund $30 \mathrm{~km}$ immer wieder abbrechen. Es erfolgten weitere Manipulationsversuche 
sowie eine Kryotherapie der unteren LWS und beim vierten Versuch hörten Arzt und Patient ein Knacken im Bereich des linken Beckens.

Nach dieser Behandlung fühlte sich der Athlet besser und er begann wieder sein Lauftraining zu forcieren. Da der Ironman-Triathlon kurz vor der Türe stand und der Triathlet noch ein Trainingsdefizit im Laufen zu korrigieren hatte probierte er möglichst schnelle Bahnläufe zu absolvieren. Dazu nahm er von einem früheren Unfall noch alte Schmerzmittel die er in regelmässigen Abständen einnahm. Am Abend führte er jeweils die erlernten Heimübungen zur Selbstbehandlung bei einem SIG-Problem durch. Ebenso führte er weitere Übungen durch die er über das Internet fand.

Da mehrere Manipulationen, Heimübungen, sowie die regelmässige Einnahme von Schmerzmitteln (150 mg Voltaren ${ }^{\circledR}, 2000$ mg Panado ${ }^{\circledR}$ ) keine Verbesserung brachten und die Laufeinheiten immer bei 30-35 km wegen Schmerzen im Bereich des linken SIG abgebrochen werden mussten meldete sich der Athlet wieder beim Hausarzt. Er berichtete auch dass das Gefühl im Bereich des hinteren Beckenkamms links vermindert sei. Wegen progredienter und therapieresistenter Schmerzen im Bereich des SIG links wurde ein MRI des Beckens durchgeführt. Dieses zeigte eine frische Fraktur des linken os ilium (Abbildung 1) mit Einstrahlung in das SIG links (Abbildung 2) und ausgedehnten ödematösen Veränderungen ossär sowie in den umgebenden Weichteilen im Bereich des os ilium. Die Fraktur verlief horizontal durch das os sacrum und das SIG und zog sich dann weiter in das os ilium. Aufgrund der Fraktur musste der Triathlet auf den Start am Wettkampf im Sommer verzichten. Der Wettkampf war eigentlich geplant um sich für einen wichtigen Wettkampf im Oktober auf dem amerikanischen Kontinent zu qualifizieren.

\section{Diagnosen}

Fraktur des linksseitigen os ilium mit Einstrahlung in das linke Sakroiliakalgelenk

\section{Kommentar}

Wir beschreiben einen Ironman-Triathleten mittleren Alters mit über 20 Jahren Wettkampferfahrung im Triathlon der sich eine Fraktur im Bereich der linken Beckenschaufel mit Einstrahlung in das linke SIG zuzog wobei sich die Fraktur klinisch als hypomobiles SIG mit Schmerzen bei Tempoläufen zeigte. 
Der Sportler wurde mit dieser Diagnose konfrontiert und es stellte sich die Frage wie so ein Bruch zustande gekommen sein könnte. Insbesondere da der Radiologe ausdrücklich darauf hinwies dass die Fraktur frisch war, horizontal durch das SIG zog mit einem Ausläufer in die linke Beckenschaufel.

Der Patient zog sein Trainingstagebuch zu Rate und hatte notiert dass er wenige Wochen vor der ersten Arztkonsultation einen Sturz durch Ausrutschen in einer Pfütze beim Laufen erlitten hatte. Er spürte dabei einen heftigen Schmerz an der Vorderseite des linken Oberschenkels sowie einen ebenso heftigen Schmerz im Bereich des Beckens auf der linken Seite. Bedingt durch die Schmerzen am Oberschenkel und im Kreuz musste er sein Lauftraining ein paar Tage pausieren und er bemerkte im Verlauf einen Bluterguss sowie eine Verformung des Oberschenkels.

Im klinischen Untersuch zeigte sich ein kompletter Abriss der distalen Sehne des $m$. rectus femoris links. Der Sportler war aber in keiner Weise in Alltag oder Training eingeschränkt und nahm diese Verletzung gar nicht gross wahr.

Es ist davon auszugehen dass sich der Triathlet beim Sturz nebst dem Sehnenabriss am ventralen Oberschenkel eine Fissur oder kleine Fraktur im Bereich des SIG links dorsal zugezogen hatte und durch das anhaltende Training gar nie eine Frakturheilung eintreten konnte. Da im MRI eine frische Fraktur ohne Zeichen einer Heilung nachweisbar wir ist davon auszugehen dass durch die mehreren Manipulationen aus einer Fissur eine Fraktur entstand, zumal bei einer der Manipulation ein deutliches Knacken zu hören war. Anamnestisch gab der Patient auch an im Verlauf nach dieser Manipulation einen Schmerz im Bereich der Gesässmuskulatur verspürt zu haben sowie ein vermindertes Gefühl am Beckenkamm auftrat. Dieser Verlauf würde auch für ein zweizeitiges Geschehen mit einer frischen Fraktur sprechen.

Eine Möglichkeit für die Erklärung dass dieser Ironman-Triathlet die Schmerzen relativ lange und gut tolerieren konnte ist die hohe Schmerztoleranz bei Sportlern. Bei Triathleten ist bekannt dass sie gegenüber der Normalpopulation eine höhere Schmerztoleranz und eine effizientere Schmerzmodulation aufweisen [1]. Unser Triathlet bestreitet seit über 20 Jahren Ironman-Triathlons und hat schon über 60 Wettkämpfe erfolgreich gefinisht.

Die vom Patienten geschilderte Gefühlsverminderung im Bereich des hinteren Beckenkamms dürfte durch die ausgedehnte ödematöse Ausdehnung in die 
Weichteile bedingt sein. Dreyfuss et al. [2] erwähnen dass eine Affektion des SIG zu einer Schmerzempfindung im Bereich der spina iliaca posterior superior führt.

\section{Wie kommt es zu einer Blockade des SIG}

In der klinischen Untersuchung wurde ein hypomobiles SIG nachgewiesen. Das SIG verbindet das os ilium mit dem os sacrum und bildet eine Amphiarthrose, also ein straffes Gelenk mit geringer Beweglichkeit und mit maximal $10^{\circ}$ Gelenkspiel. Die Beweglichkeit des SIG ist aufgrund seiner ausgeprägten ligamentären Sicherung sehr stark eingeschränkt. Die Gelenkflächen zwischen os ilium und os sacrum haben eine Bumerang-bis C-förmige Gestalt und befinden sich zwischen dem 1. und 3. Sakralwirbel. Sie bestehen aus einem oberen und einem unteren Pol, deren Umschlagstelle sich etwa in Höhe des 2. Sakralwirbels (Teil des os sacrum) befindet. Zwischen oberen und unteren Pol befindet sich eine Knickstelle, deren Winkel 100$120^{\circ}$ beträgt [3].

Eine SIG-Blockade entsteht dann, wenn das ohnehin straffe Gelenk in seiner Beweglichkeit eingeschränkt ist oder wenn die Stellung der Gelenkflächen zueinander von der Norm abweicht. Bei einer Lockerung der Bänder wie etwa bei einer Fehlbelastung kann es zu einem Verkannten bis Verhaken der Gelenkflächen kommen. Dadurch wird die auch sonst nur geringe Gelenkbeweglichkeit nahezu aufgehoben. Allerdings ist das Konzept des "blockierten" SIG pathogenetisch nicht klar definiert ist und unterliegt auch einer Kontroverse.

Eine SIG-Blockade kann sowohl bei Männern als auch bei Frauen in jedem Alter auftreten. Etwa 70\% der Bevölkerung leiden mindestens einmal im Leben unter einer SIG-Blockade. Das SIG ist in etwa 15-30\% aller Fälle mit Schmerzen im Bereich der Kreuzregion ursächlich [3, 4].

\section{Ursachen für eine SIG-Blockade}

Häufige Ursachen für eine Blockierung des SIG können schweres Heben, Überbeanspruchung beim Sport, extreme Laufbelastungen, Überdehnung der Bänder oder ungewöhnliche Bewegungen wie ein ungewollter Tritt ins Leere beim Stolpern auf der Treppe sein. Tabelle 1 stellt mögliche Ursachen für eine Problematik des SIG zusammen. Auch monotone Arbeiten oder ungünstige Körperhaltungen können zu einer SIG-Blockadeführen. Dies kann auch bei einem Verhebetrauma durch ruckartiges Anheben eines Gegenstandes vorkommen. Natürlich können auch 
Unfälle wie etwa ein Sturz mögliche Auslöser sein. In selteneren Fällen kann das SIG-Syndrom auch aus einer entzündlichen Situation heraus entstehen.

\section{Symptome bei einer SIG-Blockade}

Bei Veränderungen der Gelenkfläche (zum Beispiel durch Abnutzungsprozesse oder Erkrankungen, die mit einer Entzündung des SIG einhergehen) sowie der umgebenden Weichteile einschließlich der Bänder kann es zu einer Blockade des SIG kommen. Leitsymptom einer Blockade des SIG ist der lumbale Rückenschmerz bzw. ein Schmerz über dem os sacrum mit Ausstrahlung zur Seite und ins Gesäß, manchmal auch Oberschenkel bis Knie oder auch nur in die Leiste. Tabelle 2 stellt eine Liste von Symptomen zusammen die auf eine Problematik des SIG hinweisen könnten. Typischerweise verschlimmert sich der Schmerz beim Sitzen (höhere Belastung) und verbessert sich bei Mobilisation (Gehen). Betroffene klagen über einen ziehenden Schmerz, den sie verstärken können, wenn sie den Oberkörper stark nach vorne beugen oder das Bein der betroffenen Seite nach außen spreizen, wie etwa in Form des Schneidersitzes. Einige Betroffene wachen nachts durch Schmerzen auf und müssen sich in eine andere Position legen, um die Schmerzen zu lindern. Wenn zusätzlich eine Instabilität des SIG besteht, kann man direkt nach dem Aufstehen aus dem Sitzen ein leichtes Knacken im SIG wahrnehmen, das sich meist aber nach einigen Schritten wieder gibt. Nebst einer klassischen SIGProblematik können auch eher seltene Ursachen zu Schmerzen im Bereich des SIG führen (siehe Tabelle 3).

\section{Diagnostische Möglichkeiten}

Selbst wenn eine SIG-Problematik relativ häufig ist, so ist die korrekte klinische Diagnose eines blockierten SIG relativ schwierig [5], weil die Schmerzen oft nicht dem SIG zugeordnet werden können [6]. Zur genauen Diagnose stehen für dieses Krankheitsbild keine der bekannten bildgebenden Verfahren zur Verfügung. Über klinische Tests [3] kann festgestellt werden, ob ein SIG-Syndrom vorliegt. Tabelle 4 stellt die wichtigsten Tests zusammen, wobei die Tabelle nicht vollständig ist. Die Spezifität dieser klinischen Tests ist allerdings beschränkt. Bei mehreren positiven Tests ist die Wahrscheinlichkeit besser für die Identifikation einer SIG-Pathologie, sie erlauben jedoch nicht ein „blockiertes" SIG von einer anderen SIG-Pathologie zu unterscheiden. Das Mennel-Zeichen ist zum Beispiel typischerweise bei einer SIGArthritis positiv. Der Einsatz von diagnostischen Infiltrationen wird sehr kontrovers beurteilt $[3,4,7]$. 


\section{Therapieoptionen}

Therapeutische Möglichkeiten bei einem blockierten SIG sind Mobilisation, Manipulation, sowie der Einsatz von Schmerzmitteln [4]. In seltenen Fällen kann eine Infiltration mit Kortison in den Bandapparat des SIG hilfreich sein [4]. Bei persistierenden Beschwerden können eine thermische Denervation des Gelenks [8] sowie eine Versteifung in Betracht gezogen werden [6].

\section{Das SIG beim Sportler}

Während sportlicher Aktivität muss das Gelenk hohe Belastungen aushalten und deshalb kommt es bei Sportlern häufiger zu einer Dysfunktion des SIG als bei Inaktiven [5]. Eine Fraktur im Bereich des SIG ist relativ selten und betrifft meist isoliert das os ilium [9-11] oder das os sacrum [12-14]. Es ist auch ein Fall eines 21jährigen Leichtathleten beschrieben, der über Beschwerden im Bereich der dorsalen Oberschenkelmuskulatur und des SIG klagte. Die Schmerzen traten nur bei schnellem Laufen und bei Sprüngen auf. Die klinische Untersuchung war nicht eindeutig und aufgrund des protrahierten Verlaufs sowie der Vorgeschichte mit einer Colitis ulcerosa wurde ein MRI gemacht das eine Sacroiliitis zeigte [15]. Isolierte Frakturen des os ilium sind bei Sportlern extrem selten und dabei handelt es sicher eher um Frauen mit einer frühzeitigen Osteoporose bei einer Essstörung [9-11].

\section{Überlastungsschäden und Unfälle bei Triathleten}

Bei diesem Triathleten mittleren Alters liegt als Ursache für seine Beschwerden die seltene Ursache einer Fraktur im Bereich des SIG vor. Bei Triathleten müssen wir zwischen chronischen Überlastungsschäden am Bewegungsapparat durch Training und Wettkampf sowie Frakturen in Training und Wettkampf unterscheiden [16-19]. Beschwerden am Bewegungsapparat von Überlastungsschäden bis Frakturen hängen bei Triathleten mit verschiedenen Faktoren wie Alter, Geschlecht, Body Mass Index, Leistungsvermögen, Erfahrung und Trainingsvolumen zusammen [16, 17, 20, 21]. Beim Training liegt ein U-förmiger Zusammenhang zwischen Trainingsvolumen und Verletzung vor. Das Risiko für eine Verletzung ist bei Triathleten mit sehr geringem und wieder mit sehr hohem Trainingsvolumen viel wahrscheinlicher [21]. Bezüglich Alter und Fitnesslevel zeigten sich bei älteren Athleten eher Frakturen während bei Elite-Athleten eher Prellungen und Schürfungen sowie Schäden an Sehnen und Muskeln zu verzeichnen sind [17].

Bei den drei Disziplinen scheint die Verletzungsanfälligkeit im Training sehr unterschiedlich zu sein [16, 20, 22-24]. Triathleten verbringen einen grossen Teil 
ihres Trainings mit Radfahren und Laufen wobei die meisten Überlastungsprobleme beim Laufen auftreten [20, 23] und höchst selten beim Schwimmen [24]. Bei

Finishern an einem Triathlonwettkampf berichteten gegen 50\% der Athleten über ein Unfallereignis im Training das zu einem Unterbruch von mindestens einem Tag führte wobei $70 \%$ der Verletzungen durch das Laufen verursacht waren [16]. Im Training sind es meist Überlastungsschäden und kleiner Verletzungen wie Schürfungen oder Distorsionen, aber praktisch nie Frakturen [22, 24].

Bei der Lokalisation der Überlastungsschäden treten etwa ein Viertel der Probleme an Unterschenkel, Knie und unterem Rücken auf [20, 22]. In einer Studie trat während einer Wettkampfsaison in fast 40\% aller Triathleten mindestens eine Verletzung auf wobei die untere Extremität und der Rücken am häufigsten betroffen waren. In rund $40 \%$ der Verletzungen war es eine Überlastungsproblematik wobei diese in zwei Drittel der Fälle beim Laufen auftrat [20].

Im Wettkampf treten Verletzung eher während dem Laufen als während dem Radfahren auf [18, 20]. In einer Wettkampfserie mit über 10‘000 Startern wurden gegen $60 \%$ der Verletzungen an der unteren Extremität nachgewiesen, darunter auch fünf Frakturen. Der Fitnesslevel, das Alter und die Wettkampfdistanz haben den grössten Einfluss auf das Unfallrisiko bei einem Triathlonwettkampf [18]. In einer Studie trat während einer Wettkampfsaison in fast $40 \%$ aller Triathleten mindestens eine Verletzung auf wobei die untere Extremität und der Rücken am häufigsten betroffen waren. In rund 40\% der Verletzungen war es eine Überlastungsproblematik wobei diese in zwei Drittel der Fälle beim Laufen auftrat [20].

Eine mögliche Ursache für die Fraktur bei diesem männlichen Triathleten mittleren Alters könnte ein protrahierter Ermüdungsbruch sein, denn Ermüdungsbrüche können auch bei männlichen Triathleten mittleren Alters auftreten [25-27]. So wurde etwa der Fall eines 43-jährigen Triathleten beschrieben der sich auf der Laufstrecke an einem halben Ironman eine Stressfraktur des Femurs zuzog [25, 27]. Auch bei einem jüngeren Triathleten konnte eine Ermüdungsfraktur des Calcaneus nachgewiesen werden [27]. Bereits 1988 beschrieben Sell und Konermann [26] den Fall eines Triathleten, der während eines Rennens eine Stressfraktur in der proximalen Tibia entwickelte, nachdem er bereits zwei Stressfrakturen in den Metatarsalia sowie eine Stressfraktur in der distalen Tibia entwickelt hatte. 


\section{Verlauf im aktuellen Fall}

Der Triathlet wurde angewiesen für 12 Wochen auf Belastungen des Beckens zu verzichten. Da Laufen zu schmerzhaft war aber Radfahren und Schwimmen problemlos ging konnte der Triathlet in zwei von drei Disziplinen weiter trainieren und suchte sich eine Alternative für einen Wettkampf im Oktober. Er startete rund 12 Wochen nach der Diagnose der Fraktur an einem anderen Wettkampf auf dem amerikanischen Kontinent und konnte beim Rennen seine Kategorie mit neuem Streckenrekord gewinnen. Beim Schwimmen und Radfahren erzielte er so schnelle Abschnittszeiten wie noch nie in seinem Leben während er beim Laufen deutlich einbüsste. Die schnellen Zeiten in Schwimmen und Radfahren dürften durch das verhältnismässig hohe Trainingsvolumen erklärt werden. 


\section{Key messages}

- Die Dysfunktion des Sakroiliakalgelenks als hypomobiles Gelenk ist eine relativ häufige Erkrankung in der allgemeinen Bevölkerung und noch häufiger beim aktiven Menschen.

- Die Diagnose einer Dysfunktion des Sakroiliakalgelenks basiert auf der klinischen Untersuchung wobei in unsicheren Fällen eine diagnostische Infiltration des Gelenks in Betracht gezogen werden kann.

- Die Therapie der Dysfunktion des Sakroiliakalgelenks ist konservativ mit Mobilisation, Manipulation und Einsatz von Schmerzmitteln.

- Eine Fraktur im Bereich des os sacrum und/oder des os ilium muss bei einem sportlich aktiven Menschen mit Schmerzen im Bereich des Sakroiliakalgelenks in Betracht gezogen werden. 


\section{Zusammenfassung}

Wir berichten über einen 52-jährigen Ironman-Triathleten der über länger anhaltende Schmerzen im Bereich des linken Gesässes klagte. Therapeutische Bemühungen bei Verdacht auf ein blockiertes Sakroiliakalgelenk bleiben erfolglos. Ein MRI des Beckens zeigte dann eine Fraktur des linken os ilium. Aufgrund der Bildgebung gehen wir von einem zweizeitigen Geschehen aus mit Frakturbildung nach Manipulation.

\section{Schlüsselwörter}

Gelenk - Becken - Sportler - Fraktur 


\begin{abstract}
We report the case of a 52-year Ironman triathlete who complained of prolonged pain in the left buttock. Therapeutic efforts in the suspected blocked sacroiliac joint were unsuccessful. An MRI of the pelvis then showed a fracture of the left iliac wing. We assume that manipulation of the sacroiliac joint led to the fracture of the iliac wing.
\end{abstract}

\title{
Key words
}

Joint - Pelvis - Athlete - Fracture 
Korrespondenzadresse

Prof. Dr. med. Beat Knechtle

Facharzt FMH für Allgemeinmedizin

Gesundheitszentrum St. Gallen

Vadianstrasse 26

9001 St. Gallen

Telefon $\quad+41(0) 712269300$

Telefax $\quad+41$ (0) 712269301

E-Mail beat.knechtle@hispeed.ch 


\section{Literaturverzeichnis}

1. Geva N, Defrin R. Enhanced pain modulation among triathletes: A possible explanation for their exceptional capabilities. Pain. 2013; 154(11): 2317-23.

2. Dreyfuss P, Dreyer SJ, Cole A, Mayo K. Sacroiliac joint pain. J Am Acad Orthop Surg. 2004; 12(4): 255-65.

3. Foley BS, Buschbacher RM. Sacroiliac joint pain: Anatomy, biomechanics, diagnosis, and treatment. American Journal of Physical Medicine and Rehabilitation. 2006; 85(12): 997-1006.

4. Vanelderen P, Szadek K, Cohen SP, De Witte J, Lataster A, Patijn J, et al. 13. Sacroiliac Joint Pain. Pain Practice. 2010; 10(5): 470-8.

5. Brolinson PG, Kozar AJ, Cibor G. Sacroiliac joint dysfunction in athletes. Curr Sports Med Rep. 2003; 2(1): 47-56.

6. Daum WJ. The sacroiliac joint: An underappreciated pain generator. American Journal of Orthopedics. 1995; 24(6): 475-8.

7. Cohen SP. Sacroiliac joint pain: A comprehensive review of anatomy, diagnosis and treatment. Anesthesia and Analgesia. 2005; 101(5): 1440-53. 8. Petron DJ, Prideaux CC, Likness L. Interventional spine procedures in athletes. Current Sports Medicine Reports. 2012; 11(6): 335-40.

9. Amorosa LF, Serota AC, Berman N, Lorich DG, Helfet DL. An isolated iliac wing stress fracture in a marathon runner. American journal of orthopedics (Belle Mead, NJ). 2014; 43(2): 74-7.

10. Battaglia M, Guaraldi F, Vannini F, Vanel D, Giannini S. Unusual superomedial iliac fatigue stress fracture. Skeletal Radiol. 2012; 41(1): 103-6.

11. Haun DW, Kettner NW, Yochum TR, Green RL. Sacral Fatigue Fracture in a Female Runner: A Case Report. Journal of Manipulative and Physiological Therapeutics. 2007; 30(3): 228-33.

12. Alsobrook J, Simons SM. Sacral stress fracture in a marathon runner. Current Sports Medicine Reports. 2007; 6(1): 39-42.

13. Kahanov L, Eberman L, Alvey T, True J, Yeargin B. Sacral stress fracture in a distance runner. Journal of the American Osteopathic Association. 2011; 111(10): 585-91.

14. Tzoanos G, Tsavalas N, Manidakis N, Karantanas A. Sacral fatigue fracture in an amateur soccer player. Case Rep Med. 2013; 2013: 985310.

15. Miller TL, Cass N, Siegel C. Ankylosing spondylitis in an athlete with chronic sacroiliac joint pain. Orthopedics. 2014; 37(2): e207-e10. 
16. Collins K, Wagner M, Peterson K, Storey M. Overuse injuries in triathletes: A study of the 1986 Seafair Triathlon. The American Journal of Sports Medicine. 1989; 17(5): 675-80.

17. Egermann M, Brocai D, Lill CA, Schmitt $\mathrm{H}$. Analysis of injuries in longdistance triathletes. International Journal of Sports Medicine. 2003; 24(4): 271-6. 18. Gosling CM, Forbes AB, McGivern J, Gabbe BJ. A profile of injuries in athletes seeking treatment during a triathlon race series. American Journal of Sports Medicine. 2010; 38(5): 1007-14.

19. Rüst $\mathrm{CA}$, Knechtle $\mathrm{B}$, Knechtle $\mathrm{P}$, Rosemann $\mathrm{T}$. Atrophy of the brachialis muscle after a displaced clavicle fracture in an Ironman triathlete: Case report. Journal of Brachial Plexus and Peripheral Nerve Injury. 2011; 6(1).

20. Korkia PK, Tunstall-Pedoe DS, Maffulli N. An epidemiological investigation of training and injury patterns in British triathletes. British Journal of Sports Medicine. 1994; 28(3): 191-6.

21. Shaw $T$, Howat $P$, Trainor $M$, Maycock $B$. Training patterns and sports injuries in triathletes. Journal of Science and Medicine in Sport. 2004; 7(4): 446-50.

22. Andersen CA, Clarsen B, Johansen TV, Engebretsen L. High prevalence of overuse injury among iron-distance triathletes. British Journal of Sports Medicine. 2013; 47(13): 857-61.

23. Spiker AM, Dixit S, Cosgarea AJ. Triathlon: Running injuries. Sports Medicine and Arthroscopy Review. 2012; 20(4): 206-13.

24. Zwingenberger S, Valladares RD, Walther A, Beck H, Stiehler M, Kirschner S, et al. An epidemiological investigation of training and injury patterns in triathletes. Journal of Sports Sciences. 2014; 32(6): 583-90.

25. Larsen $\mathrm{P}$, Elsoe R, Rathleff MS. A case report of a completely displaced stress fracture of the femoral shaft in a middle-aged male athlete - A precursor of things to come? Physical Therapy in Sport. 2016; 19: 23-7.

26. Sell S, Konermann W. Triathlon--continuous stress on muscles, tendons and bones? Sportverletzung Sportschaden : Organ der Gesellschaft fur OrthopadischTraumatologische Sportmedizin. 1988; 2(3): 112-4.

27. Serrano S, Figueiredo P, Páscoa Pinheiro J. Fatigue fracture of the calcaneus: From early diagnosis to treatment. American Journal of Physical Medicine and Rehabilitation. 2016; 95(6): e79-e83. 
Tabelle 1: Mögliche Ursachen für eine SIG-Problematik

Fehlhaltungen

Fehlbelastungen

Schonhaltungen durch einseitiges Hinken und/oder Entlastungshinken

Wiederkehrende unphysiologische Bewegungen im Alltag

Beckenschiefstand durch Beinlängendifferenz

Hyperflexibilität des Halteapparates

Bewegungsmangel

Verhebetrauma durch Heben von zu schweren Gewichten

Sturz auf das Gesäss

Ins 'Leere' treten

Stolpern bei bereits labilem SIG

Plötzliche Stoppbewegung

Muskuläre Dysbalance

Hoher Muskeltonus aufgrund von Stress und nicht ausreichender Entspannung 
Tabelle 2: Typische Symptome bei einer SIG-Problematik

Schmerzen direkt im SIG

Diffuse Kreuzschmerzen

Ausstrahlender Schmerz in das Gesäss

Ausstrahlender Schmerz in die Leiste

Ausstrahlender Schmerz in die Oberschenkelvorderseite

Ausstrahlender Schmerz in die LWS

Sensibilitätsstörungen wie Kribbeln und Ameisenlaufen

Gefühl des Hinkens oder Klemmens im Hüftgelenk

Bewegungseinschränkung im Hüftgelenk

Gefühl der Instabilität des Beckens und des unteren Rückens

Schmerzauslösung oder -verstärkung durch starkes Vor- oder Rückwärtsbeugen

Schmerzverstärkung nach längerem Sitzen

Schmerzlinderung durch Kippen des Beckens nach dorsal

Schmerzen im Sitzen, Stehen, oder Liegen

Knieschmerzen 
Tabelle 3: Mögliche Differentialdiagnosen für eine SIG-Problematik

Beckentorsion

Piriformis-Syndrom

Triggerpunkte in der Glutealmuskulatur

Lumbale Diskushernie

Morbus Bechterew

Sacroiliitis

Lyme-Borreliose

Coxarthrose

Osteoporose-bedingte Fraktur

Primärtumor im Becken

Metastasen

Psychisch bedingte Rückenschmerzen

Psychogene Anspannung durch Angst, Ärger, Stress, oder Krankheit

Bei der Frau Lockerung des Bänderapparates in der Schwangerschaft

Bei der Frau durch Schwangerschaft und Geburt 


\section{Tabelle 4: Diagnostische Tests}

Vorlaufphänomen: Die spina iliaca posterior superior wird im Stehen beidseits getastet. Man vergleicht diese Position der spina iliaca posterior superior im Stehen mit der Position nach einer Beugung des Oberkörpers. Von einem positiven Vorlaufphänomen spricht man, wenn durch ein blockiertes SIG die spina iliaca posterior superior der blockierten Seite mit dem Kreuzbein nach oben gezogen wird.

Spine-Test: Bei nicht blockiertem SIG sinkt die spina iliaca posterior superior beim Anheben des gleichseitigen Beines bis $90^{\circ}$ im gebeugtem Knie ca. 1-2 cm nach unten.

Maigne-Test: Der Patient liegt auf dem Rücken, stellt ein Bein angewinkelt auf und lässt es nach aussen fallen. Liegt eine Veränderung des SIG vor, ist diese Bewegung einseitig nur beschränkt möglich.

Mennel-Zeichen: In Bauchlage wird das os sacrum fixiert und das gestreckte Bein angehoben und in eine Hüftüberstreckung gebracht. Liegt eine Blockierung des SIG vor, werden dadurch Schmerzen ausgelöst. 


\section{Legende der Abbildungen}

Abbildung 1: Fraktur im os ilium links (horizontal verlaufend, T1-gewichtet, sagittal). Der Pfeil zeigt den Frakturverlauf.

Abbildung 2: Fraktur im os ilium links in Längsrichtung entlang der Beckenschaufel mit Einstrahlung in das Sakroiliakalgelenk (T2-gewichtet, transversal). Der Pfeil zeigt den Frakturverlauf. 


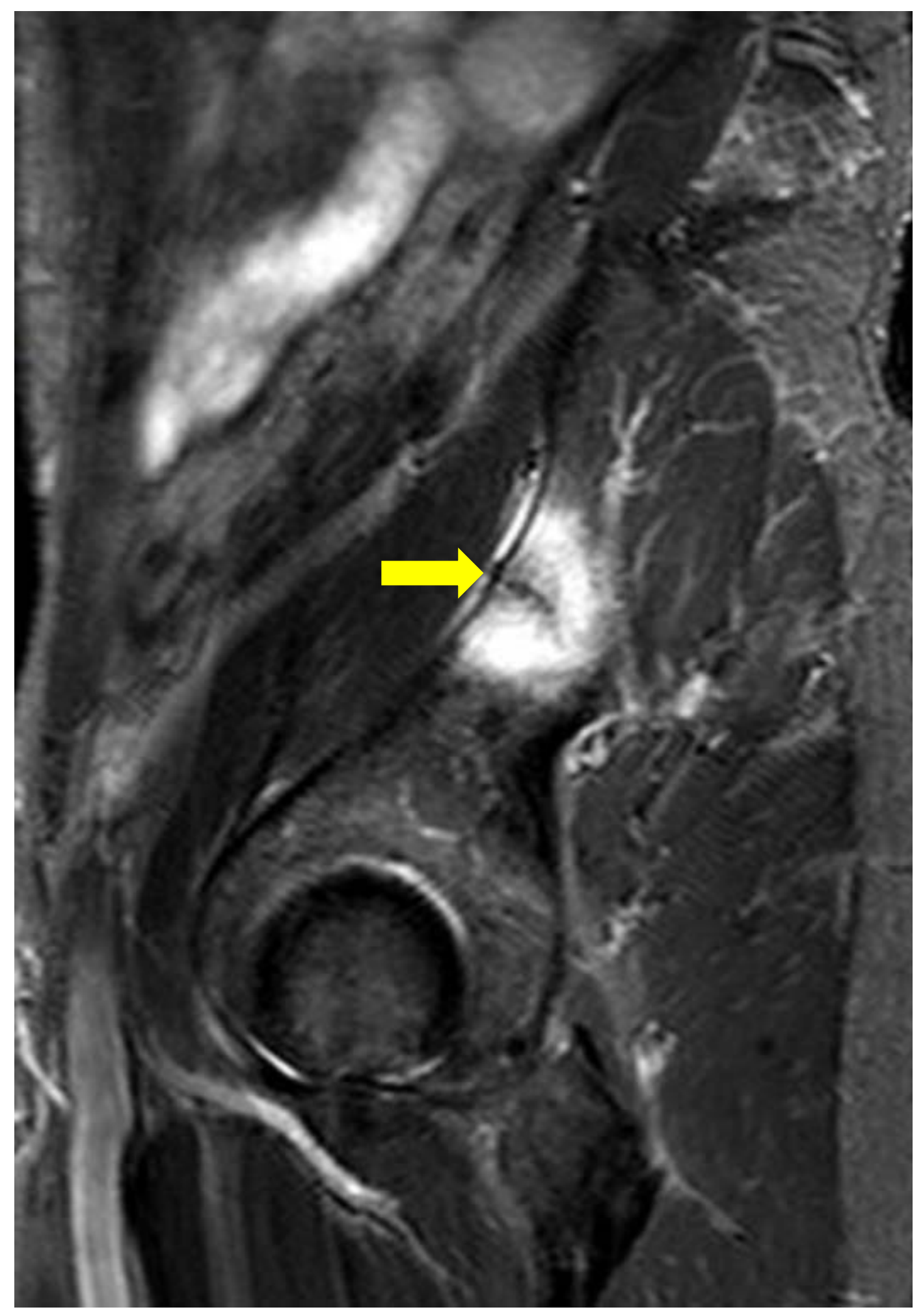

Abbildung 1 


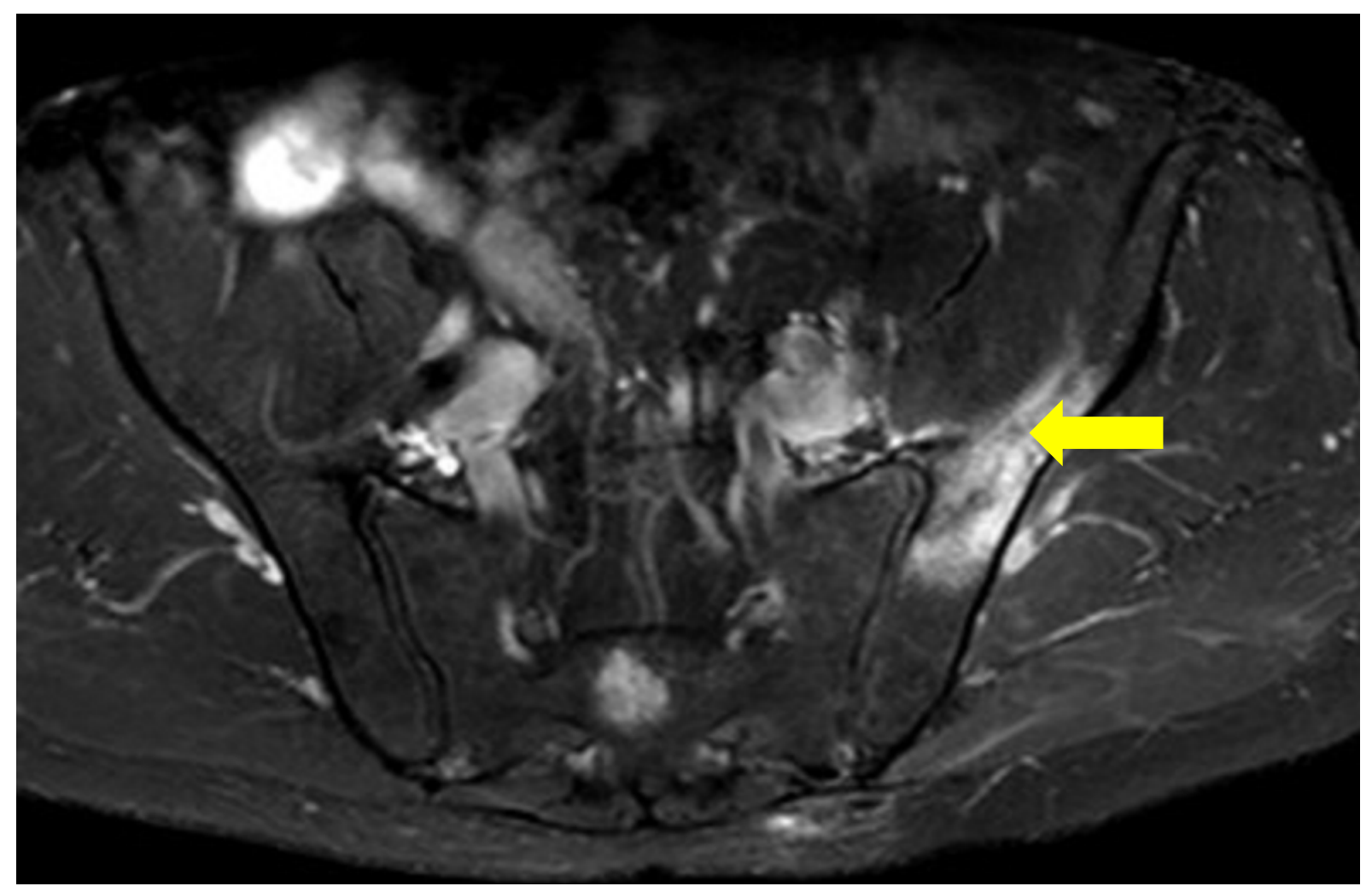

Abbildung 2 